\section{Les glomérulonéphrites extramembraneuses : un modèle d'étude des antigènes néphritogènes}

Les glomérulonéphrites extramembraneuses sont caractérisées par la présence de dépôts immuns granuleux situés à la face externe (épithéliale) de la membrane basale du capillaire glomérulaire. Le modèle expérimental de la glomérulonéphrite induite chez le rat par l'immunisation à l'aide de préparations de bordure en brosse tubulaire a permis de détecter plusieurs cibles antigéniques néphritogènes, dont la plus importante est une glycoprotéine de $330 \mathrm{kDa}$ associée à la clathrine des puits mantelés (coated pits). Chez l'homme, où cette maladie est une des causes majeures de glomérulonéphrite, les cibles antigéniques sont plus diverses, d'origine glomérulaire ou extraglomérulaire. Leur caractérisation pourrait peut-être permettre, dans l'avenir, d'éliminer spécifiquement par immuno-absorption les anticorps dirigés contre des antigènes néphritogènes.

\section{Pierre Ronco \\ Monique Géniteau Édouard Van Leer Lando Allegri François Châtelet Pierre Verroust}

\section{ADRESSES}

P. Ronco: professeur à l'université Paris VI. M. Géniteau : maître de conférences à l'université Paris XI. F. Châtelet : maître de conférences à l'université Paris VI. P. Verroust: Directeur de recherches à l'Inserm. Hôpital Tenon, 4, rue de la Chine, 75970 Paris Cedex 20, France.

E.H.G. Van Leer : docteur en médecine, laboratoire de pathologie, université de Leyden, Pays-Bas. L. Allegri : docteur en médecine, clinique néphrologique, université de Parme, Parme, Italie.

TIRÉS A PART

P. Ronco.

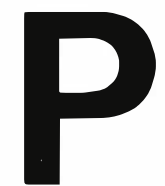

lus de vingt ans après les premières études en immunofluorescence des biopsies rénales montrant la présence de dépôts d'immunoglobulines ( $\mathrm{Ig}$ ) et de composants du complément (C') dans les glomérulonéphrites, la nature des cibles antigéniques reste encore le plus souvent inconnue. On sait que les dépôts linéaires d'Ig sont dus à des anticorps (Ac) dirigés contre le collagène IV, un composant majoritaire de la membrane basale du capillaire glomérulaire. Des études récentes ont permis de localiser le déterminant antigénique - ou épitope - dans le domaine $\mathrm{NCl}$ de la partie non-collagène de la molécule. En revanche, quand les dépôts immuns sont granuleux, situation de loin la plus fréquente, l'antigène n'est pas, habituellement, identifié. Pendant longtemps, à la suite des études sur la maladie sérique, on a cru que cette situation correspondait toujours au dépôt de complexes immuns circulants. Dans des cas exceptionnels, il a été possible d'identifier au sein de ces dépôts des antigènes microbiens ou endogènes (ADN, antigènes tumoraux, thyroïdiens...) et/ou d'éluer à partir des glomérules des Ig dirigées contre ces antigènes.

Des progrès importants dans l'analyse du contenu et de la formation des dépôts immuns ont été réalisés grâce à l'étude expérimentale des glomérulonéphrites extramembraneuses (GEM), variété de glomérulonéphrite dans laquelle les lésions se résument initialement à des dépôts granuleux denses aux électrons sur le versant épithélial du capillaire glomérulaire. Divers travaux ont montré que les dépôts extramembraneux d'Ig pouvaient résulter de la formation locale 
RÉFÉRENCES

1. Heymann W, Hackel DB, Harwood S, Wilson SGF, Hunter JLP. Production of nephrotic syndrome in rats by Freund's adjuvants and rat kidney suspensions. Proc Soc Exp Biol Med 1959; 100 : 660-4.

2. Edgington TS, Glassock RJ, Dixon FJ, Autologous immune complex nephritis induced with renal tubular antigen. I. Identification and isolation of the pathogenetic antigen. J Exp Med 1968 ; 127 : 555-72.

3. Barabas $\mathbf{A Z}$, Lannigan $R$. Induction of an autologous immune-complex glomerulonephritis in the rat by intravenous injection of heterologous anti-rat kidney tubular antibody. I. Production of chronic progressive immune-complex glomerulonephritis. $\mathrm{Br} \mathrm{J}$ Exp Pathol 1974 ; 55 : 47-55

4. Van Damme BJC, Fleuren GJ, Bakke WW, Vernier R, Hoedemaeker PHJ. Experimental glomerulonephritis in the rat induced by antibodies directed against tubular anti gens. V. Fixed glomerular antigens in the pathogenesis of heterologous immune com plex glomerulonephritis. Lab Invest 1978; 38 : 502-10.

5. Couser WG, Steinmuller DR, Stilman MM. Experimental glomerulonephritis in the isolated perfused rat kidney. J Clin Invest $1978 ; 62$ : 1275-87.

6. Feenstra K, Van De Lee R, Greben HA Arends A, Hoedemaeker PHJ. Experimenta glomerulonephritis in the rat induced by antibodies directed against tubular antigen. I The natural history : a histologic and immunohistologic study at the light microscopic and ultrastructural level. Lab Invest 1975 ; 32 . 235-42.

7. Fleuren GJ, Van De Lee R, Greben HA, Van Damme BJC, Hoedemaeker PHJ. Experimental glomerulonephritis in the rat induced by antibodies directed against tubula antigens. IV. Investigations into the pathogenesis of the model. Lab Invest 1978 ; 38 : 496501 .

8. Bertani T, Nolin L, Foidart J, Vandewalle $A$, Verroust $P$. The effect of puromycin on subepithelial deposits induced by antibodies directed against tubular antigens : a quantitative study. Eur J Clin Invest 1979 ; 9 : 465 72.

9. Neale TJ, Couser WG, Salant DJ, Lowenstein LM, Wilson CB. Specific uptake of Heymánn's nephritic kidney eluate by rat kidney. Lab Invest $1982 ; 46: 450-3$.

10. Neale TJ, Wilson CB. Glomerular antigens in Heymann's nephritis : reactivity of eluted and circulating antibody. J. Immunol 1982 ; 128 : 323-30

11. Kerjaschki D, Farquhar MG. The patho genic antigen of Heymann nephritis is membrane glycoprotein of the renal proxima tubule brush border. Proc Natl Acad Sci USA 1982 ; 79 : 5557-61.

12. Kerjaschki D, Farquhar MG. Immunocytochemical localization of the Heymann nephritis antigen (gp 330) in glomerular epithelial cells of normal Lewis rats. J Exp Med $1983 ; 157: 667-86$

13. Ronco P, Allegri L, Verroust P. Produc tion of monoclonal antibodies to rat brush border antigens. Eur J Clin Invest 1983; 13 : 13 (abstr.). (in situ) dans la paroi du capillaire glomérulaire d'un complexe immun entre un Ac libre circulant et un antigène glomérulaire intrinsèque ou « planté », irrégulièrement distribué. De plus, ils ont permis d'identifier plusieurs antigènes glomérulaires «néphritogènes gènes », c'està-dire gènes pouvant servir de cibles à un conflit immunologique.

Dans la première partie de cet article, nous exposons les constatations faites dans un modèle expérimental classique de GEM, la glomérulonéphrite de Heymann (GNH), qui ont conduit à l'identification de l'antigène cible, une protéine de $330 \mathrm{kDa}$ exprimée sur et synthétisée par les cellules épithéliales glomérulaires. Nous montrons également que d'autres antigènes membranaires synthétisés par ces mêmes cellules peuvent être impliqués dans la formation de dépôts immuns dont la cinétique est différente. Dans la seconde partie, nous discutons l'extrapolation des résultats expérimentaux aux GEM humaines.

\section{Glomérulonéphrites extramembraneuses expérimentales}

La glomérulonéphrite de Heymann.

- Premières études : le rôle des complexes immuns circulants (CIC). En 1959, Heymann et al. [1] décrivent chez le rat un modèle de GEM induite par immunisation avec des préparations de bordure en brosse du tube contourné proximal. En quelques semaines, les animaux développent une protéinurie importante, un syndrome néphrotique et des lésions glomérulaires proches des GEM humaines. En 1968, Edgington et al. [2] suggèrent que la maladie est secondaire au dépôt, dans les glomérules, de complexes antigènes/anticorps faisant intervenir un antigène tubulaire présent dans la circulation et les anticorps correspondants. Cette conclusion repose essentiellement sur deux observations. D'une part, la maladie peut être induite par des préparations membranaires (dénommées FxlA) dérivées de la bordure en brosse (BB) du tubule rénal proximal à partir desquelles il est possible de purifier une préparation néphritogène RTE $\alpha 5$. D'autre part, les Ig éluées à partir des glomérules de rats néphritiques (de même que les anticorps anti-FxlA et les anticorps anti RTE $\alpha 5$ ) réagissent avec la bordure en brosse de reins normaux ou pathologiques, avec les glomérules de reins néphritiques, mais les techniques immunomorphologiques utilisées à cette époque n'ont pas permis de mettre en évidence une réactivité avec les glomérules normaux.

- La glomérulonéphrite de Heymann passive: formation locale des dépôts immuns. La mise au point d'un modèle de glomérulonéphrite de Heymann (GNH) passive [3] c'està-dire induite par injection parentérale d'anticorps hétérologues antibordure en brosse (anti-BB) amène à penser que les dépôts extramembraneux d'Ig peuvent être formés en l'absence de complexe immun circulant. En effet, Van Damme et al. [4] et Couser et al.[5], utilisant des modèles de reins perfusés ex vivo ou de reins isolés, induisent des dépôts glomérulaires d'Ig dans des conditions expérimentales où le rôle de complexes immuns circulants peut être exclu puisqu'il n'y a pas d'antigène circulant. Ces observations suggèrent que les anticorps anti-bordure en brosse réagissent directement avec un antigène glomérulaire. Elles constituent des arguments importants en faveur de la formation locale, in situ, des dépôts d'Ig, mais la démonstration in vitro de l'antigène cible glomérulaire, par des méthodes immunohistologiques, s'avère difficile $[6,7]$.

Au cours d'expériences utilisant des anticorps anti-FxlA purifiés par chromatographie d'affinité [8], nous avons mis en évidence une fixation fine et irrégulière d'Ig sur les sections glomérulaires et sur des cellules épithéliales glomérulaires en culture. De plus, Neale et Wilson $[9,10]$ montrent que les Ig éluées à partir de glomérules de rats ayant une GNH active, de même que les anticorps anti-RTE $\alpha 5$, réagissent in vitro avec les glomérules et induisent des dépôts glomérulaires dans un système de rein perfusé isolé. Il faut noter que, ces expériences ayant été réalisées avec des préparations d'anticorps polyclonaux, il n'était pas possible d'exclure l'intervention de deux populations d'anticorps, l'une spécifique d'un ou plusieurs anti- 

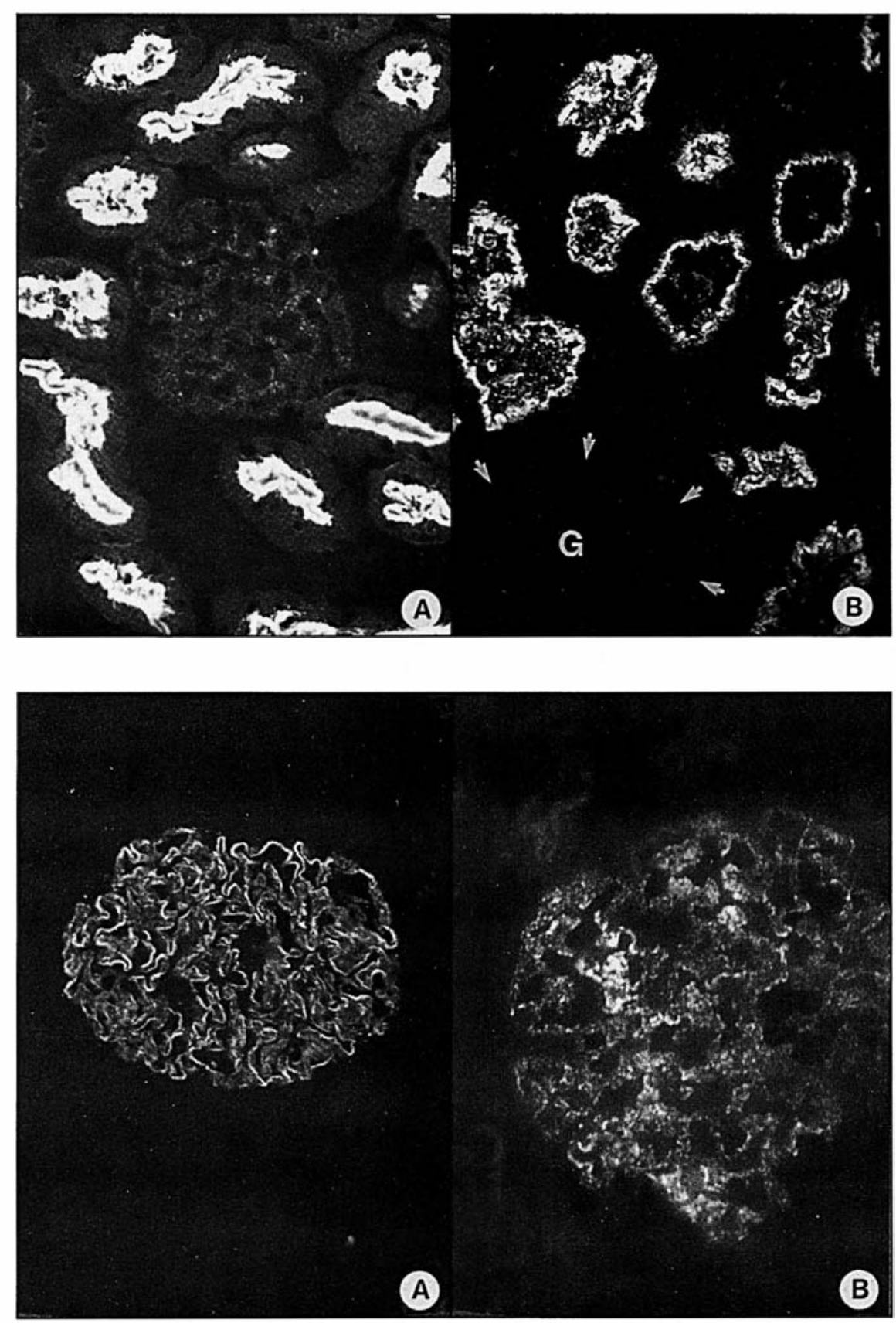

gènes tubulaires, l'autre spécifique d'un ou plusieurs antigènes glomérulaires.

- Identification de l'antigène cible: une glycoprotéine de 330 kDa. L'identification de l'antigène cible a permis d'obtenir une preuve définitive du mécanisme de formation in $m / s n^{\circ} 5$ vol. 5 , mai 89 situ des dépôts glomérulaires. Il s'agit d'une glycoprotéine de $330 \mathrm{kDa}$ initialement purifiée par Kerjaschki et al. [11, 12] par chromatographie d'affinité sur lectine de Lens Culinaris à partir de bordures en brosse solubilisées par le désoxycholate de sodium. Notre approche personnelle du problème a comporté une «dissection antigénique » de la

Figure 1. Expression de gp 330 dans le rein de rat (A) et le rein humain (B), étudiée en immunofluorescence indirecte à l'aide d'anticorps monoclonaux. Chez le rat (A), I'antigène gp 330 est abondant dans les régions intermicrovillaires de la bordure en brosse des tubes contournés proximaux; il est exprimé plus faiblement et de façon irrégulière et granuleuse dans le glomérule. Chez l'homme (B), la protéine a la même distribution dans la bordure en brosse, mais est absente du glomérule (G). (x 250).

Figure 2. Dépôts d'immunoglobulines (Ig) dans les glomérules de rats immunisés avec la protéine gp 330 purifiée (GN de Heymann active) (A) ou injectés avec des anticorps polyclonaux de lapin anti-gp 330 (GN de Heymann passive) (B). Les Ig sont détectées à l'aide d'anticorps anti-lgG de rat en (A) et antiIgG de lapin en (B). Noter la présence de dépôts très abondants pseudolinéaires dans la forme active (A) et plus fins et dispersés dans la forme passive (B). ( $\times$ 250, A ; x 400, B.)

bordure en brosse grâce à la production d'anticorps monoclonaux qui permettent d'invidualiser des antigènes présents à la fois sur cette bordure en brosse et sur le glomérule $[13,14,15]$. Nous avons ainsi produit sept anticorps qui réagissent avec une protéine de $330 \mathrm{kDa}$ identique à celle décrite par Kerjaschki et al.. La distribution tissulaire de gp 330 est 


\section{RÉFÉRENCES}

14. Ronco P, Melcion C, Geniteau M, et al. Production and characterization of monoclonal antibodies against brush border antigens of the proximal convoluted tubule. Immunology $1984 ; 53: 87-95$.

15. Ronco P, Neale TJ, Wilson CB, Galceran $M$, Verroust $P$. An immunopathological study of a $330 \mathrm{kD}$ protein defined by monoclonal antibodies and reactive with antiRTE $\alpha 5$ antibodies and kidney eluates from active Heymann nephritis. J Immunol 1986 ; 136 : 125-30.

16. Chatelet F, Brianti E, Ronco P, Roland J, Verroust P. Ultrastructural localization by monoclonal antibodies of brush border antigens expressed by glomeruli : I. Renal distribution. Am J Pathol 1986: 122 : 500-11.

17. Chatelet F, Brianti E, Ronco P, Roland J, Verrroust $P$. Ultrastructural localization by monoclonal antibodies of brush border antigens expressed by glomeruli : II. Extrarenal distribution. Am J Pathol 1986 ; 122 : 512-9.

18. Allegri L, Brianti E, Chatelet F, Manara GC, Ronco P, Verroust P. Polyvalent antigen-antibody interactions are required for the formation of electron-dense immune deposits in passive Heymann's nephritis. $\mathrm{Am}$ J Pathol 1986; 126 : 1-6.

19. Andres G, Brentjens JR, Caldwell PRB, Camussi G, Matsuo S. Biology of disease. Formation of immune deposits and disease. Lab Invest 1986 ; 55 : 510-20.

20. Camussi G, Noble B, Van Liew J, Brentjens J, Andres G. Pathogenesis of passive Heymann nephritis: chlorpromazine inhibits antibody-mediated redistribution of cell surface antigens and prevents development of the disease. J. Immunol 1986 ; 136 ; 2127-35.

21. Kerjaschki D, Miettinen A, Farquhar MG. Initial events in the formation of immune deposits in passive Heymann nephritis. J Exp Med 1987 ; 166 : 109-28.

22. Ronco P, Allegri L, Melcion C, et al. A monoclonal antibody to brush border and passive Heymann nephritis. Clin Exp Immunol 1984 ; 55 : 319-32.

23. Van Leer EHG, Moullier PH, Ronco P, Verroust P. Lymphocyte expression of a 90 $\mathrm{kDa}$ brush border antigen. Clin Exp Immunol 1987 ; 67 : 572-80.

24. Jeraj K, Vernier RL, Sisson SP, Michael AF. A new glomerular antigen in passive Heymann's nephritis. Br J Exp Pathol 1984 ; 65 : 485-98.

25. Matsuo S, Fukatsu A, Taub ML, Caldwell PRB, Andres G. Glomerulonephritis induced in the rabbit by anti-endothelial antibodies. J Clin Invest 1987; 79 : 1798-811. 26. Assmann KJM, Tangelder MM, Lange WPH, Tadema TM, Koene RAP. Membranous glomerulonephritis in the mouse. Kidney Int 1983 ; 24 : 303-12.

27. Assmann KJM, Ronco P, Tangelder $M$, Lange WPH, Verroust P, Koene RAP. Comparison of antigenic-targets involved in antibody-mediated membranous glomerulonephritis in mouse and rat. Am J Pathol 1985 ; $121: 112-22$.

28. Ronco $\mathrm{P}$, Van Leer EHG, Chatelet F, Tauc M, Verroust $P$. Brush border (BB) hydrolases expressed by glomerular epithelial cells

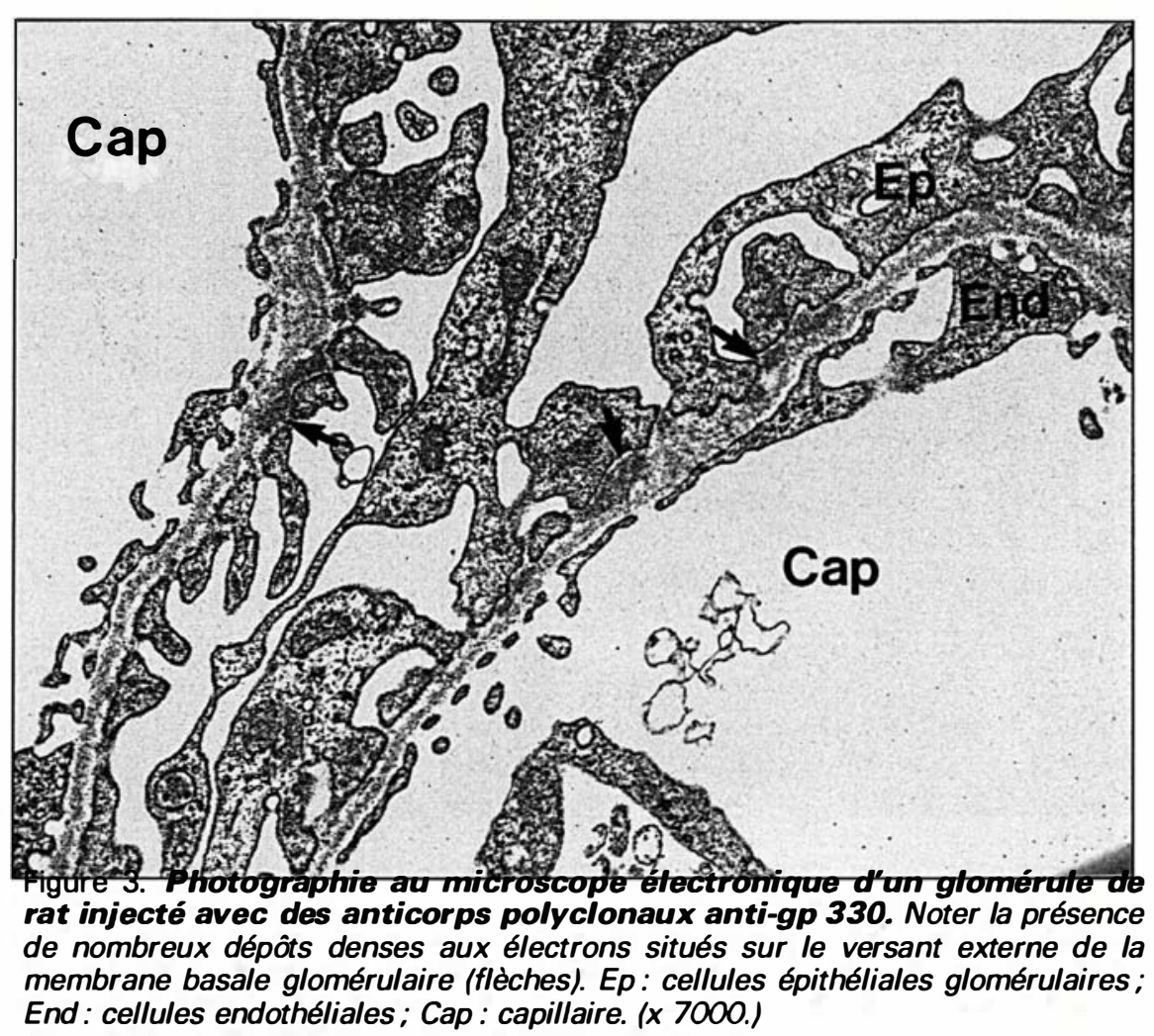

très particulière. Dans le rein [16], elle est exprimée dans les espaces intermicrovillaires de la bordure en brosse et les puits mantelés des cellules épithéliales glomérulaires (figure 1A). En dehors du rein [17], gp 330 est exprimée par les cellules épithéliales de l'épididyme, les pneumocytes II et les cellules épithéliales viscérales du sac vitellin. Ces domaines membranaires sont carartérisés par la présence de clathrine sur leur versant cytoplasmique. La localisation de gp 330 en association avec la clathrine suggère que la protéine est impliquée dans le processus d'endocytose par récepteurs. Sa distribution restreinte à certains tissus amène à penser qu'elle-même pourrait être un récepteur pour un ligand non encore identifié.

Le rôle de gp 330 dans la pathogénie de la GNH repose sur trois types d'expériences [16]. Les techniques d'immunoprécipitation montrent que les Ig éluées à partir des glomérules de rats ayant une GNH active ou passive sont spécifiques de gp 330. Il est intéressant de noter que les anticorps polyclonaux anti-
RTE $\alpha 5$ (l'antigène néphritogène isolé en 1968 par Edgington et al.) réagissent avec gp 330, ce qui indique que cette protéine était impliquée dans les expériences classiques réalisées par ces auteurs sur la GNH. En second lieu, l'immunisation active de rats avec gp 330 purifié par chromatographie d'affinité induit une GEM caractéristique (figure $2 A$ ) ainsi qu'une protéinurie. L'analyse membraneux contiennent des Ig de rat, mais aussi gp 330. Les taux d'anticorps anti-gp 330 reflètent la munisation passive de rats par des anticorps anti-gp 330 induit des dépôts extramembraneux caractéristiques (figure $2 B$ ) et une protéinurie. - Formation des dépôts denses aux électrons. L'idenfication de l'antipermet d'aborder l'analyse des mécanismes qui permettent la formation de dépôts denses aux électrons caractéristiques de la GEM à partir d'une réaction antigène-anticorps initialement localisée dans les puits manimmuno-ultrastructurale des glomérules montre que les dépôts extrasévérité de la maladie. Enfin, l'imgène cible responsable de la GNH 
telés de la cellule épithéliale glomérulaire. L'étude de la liaison in vivo des anticorps polyclonaux et monoclonaux anti-gp 330 nous a permis de faire des constatations qui permettent de mieux comprendre ces étapes [18]. Nous avons, en effet, montré que si les deux types d'anticorps sont capables de former des dépôts immuns, seuls les anticorps polyclonaux sont capables d'induire des dépôts denses aux électrons (figure 3). Ces observations rappellent celles qui avaient été faites antérieurement par le groupe d'Andres [19] dans d'autres systèmes. Elles suggèrent que la liaison d'anticorps divalents polyclonaux avec un antigène exprimé par une surface cellulaire permet la formation de complexes immuns de grande taille, hautement réticulés, qui sedétachent ensuite de la membrané cellulaire pour former les dépôts denses aux électrons. Ce processus (analysé dans [19] et schématisé sur la figure 4) implique: (1) une redistribution de l'antigène sur la surface cellulaire, ce qui requiert un cytosquelette fonctionnel [20]; (2) une élimination au moins partielle de l'antigène de la surface cellulaire, ce qui induit une synthèse accrue de gp 330 par la cellule épithéliale [19]. L'ensemble de ces expériences met en lumière l'importance des événements secondaires au processus immun initial. Il pourrait rendre compte des résultats variables concernant l'induction d'une GNH passive. Kerjaschki et al. [12] ont récemment étudié la phase initiale de formation des dépôts denses aux électrons au cours de la GNH passive. Ces travaux apportent deux éléments importants : d'une part, les dépôts immuns formés dans les minutes qui suivent l'injection d'anticorps anti-gp 330 adhèrent fortement à la membrane basale glomérulaire. Les mécanismes impliqués sont discutés mais la stabilité de la liaison suggère qu'elle pourrait être de nature covalente. D'autre part, l'analyse de sections glomérulaires sériées montre que les dépôts denses aux électrons restent, au moins dans les huit jours qui suivent l'injection d'anticorps, reliés à un puits mantelé. Le devenir des complexes immuns gp 330-anti-gp 330 - dont la majorité ne semblent pas internalisés mais forment le noyau des $\mathrm{m} / \mathrm{s} n^{\circ} 5 \mathrm{vol} .5$, mai 89 dépôts extramembraneux extracellulaires - est en opposition avec celui des complexes récepteur-ligand qui sont internalisés dans le processus d'endocytose par récepteur. Cette divergence suggère que gp 330 pourrait différer dans une partie cruciale de sa séquence des récepteurs membranaires qui se regroupent dans les puits mantelés avant leur internalisation, et suscite des efforts importants visant à cloner le gène de gp 330 . Gp 90 : un antigène impliqué dans la formation de dépôts transitoires. Au cours du processus de sélection des anticorps monoclonaux antibordure en brosse, nous avons isolé deux anticorps qui réagissent avec une protéine de $90 \mathrm{kDa}$ (figure 6) exprimée par les cellules épithéliales du tubule proximal et du glomérule $[13,22]$ (figure 5A). L'analyse de sa distribution à l'échelon ultrastructural montre qu'à l'inverse de gp 330 , gp 90 est exprimée sur toute la hauteur des microvilli et de.manière diffuse sur la surface des cellules épithéliales et des cellules endothéliales glomérulaires. En dehors du rein, gp 90 Cest exprimée par des épithéliums de transport (intestin, pôle biliaire des hépatocytes), les endothéliums capillaires et les lymphocytes [23]. Cette distribution nous a amenés à rechercher et à mettre en évidence les propriétés enzymatiques de gp 90 : il s'agit de la dipeptidyl peptidase IV (DPP IV) [28].
La cinétique des dépôts glomérulaires induits par les anticorps antigp 90 est très particulière. Alors que les anticorps anti-gp 330 forment des dépôts glomérulaires stables qui peuvent persister pendant des jours, voire des mois, les anticorps monoou polyclonaux anti-gp 90 induisent des dépôts glomérulaires transitoires: maximaux dans les quatre heures qui suivent l'injection intraveineuse, ils diminuent très rapidement et sont pratiquement non décelables après 72 heures. Ces observations ont l'intérêt de mettre en lumière le rôle crucial des propriétés de l'antigène cible dans la formation des dépôts immuns.

Le rôle éventuel joué par gp 90 en immunopathologie chez le rat dans la GNH est particulièrement difficile à étudier en raison du rôle prépondérant de gp 330 dans l'induction de la néphropathie. Les Ig éluées à partir des glomérules de rats ayant une GNH ne réagissent pas avec gp 90 . Cette observation n'exclut pas le rôle de ce système antigénique dans la maladie de Heymann. On peut, en effet, penser que des complexes immuns formés à la surface des cellules épithéliales et endothéliales ne persistent pas dans les glomérules, mais accroissent la perméabilité glomérulaire et favorisent la fixation d'anticorps anti-gp 330. Il est à cet égard intéressant de noter que le rôle de complexes formés à la surface des

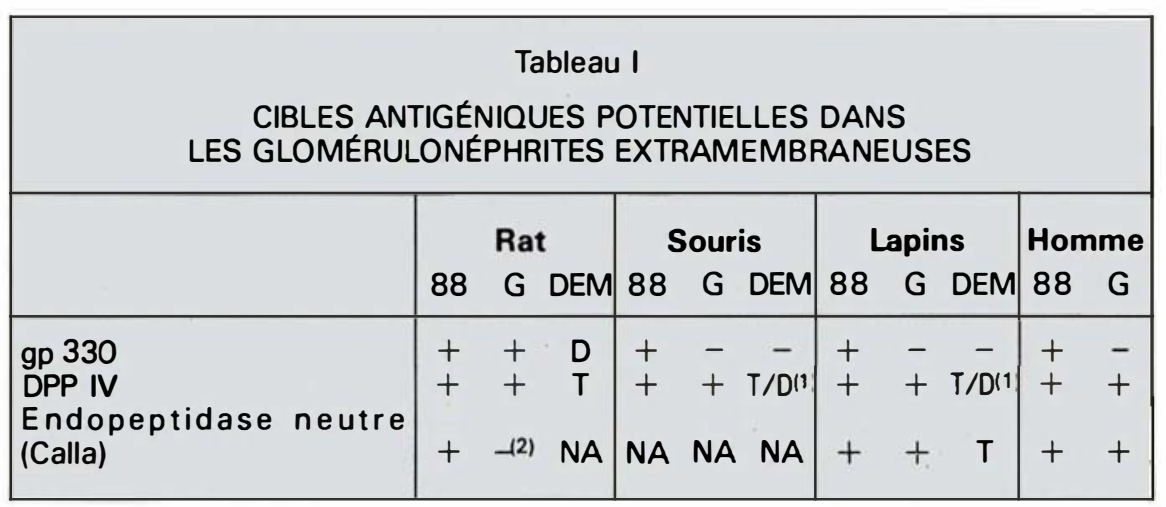

$B B$ : expression sur la bordure en brosse; $G$ : expression sur le glomérule; DEM : dépôts extramembraneux induits par l'injection de l'anticorps correspondant; $D$ : durables; $T$ : transitoires; NA : non analysé.

(1) Phase hétérologue transitoire, phase autologue durable. (2) Expression restreinte aux cellules épithéliales pariétales de la capsule de Bowman. 


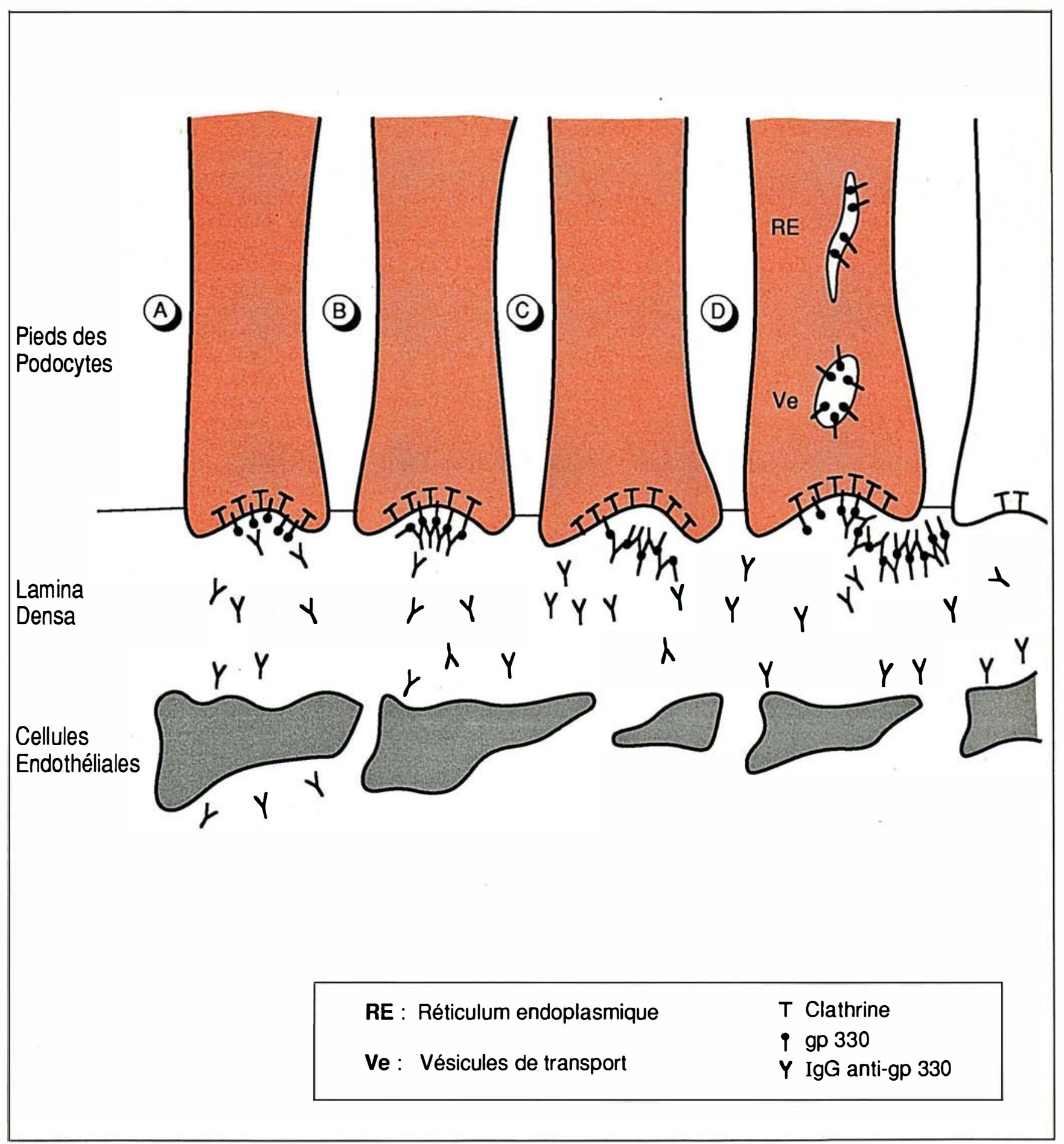

Figure 4. Représentation schématique des premières étapes de la formation des dépôts immuns dans la glomérulonéphrite de Heymann passive (d'après Kerjaschki et Farquhar). (A) Les anticorps anti-gp 330 circulants (Y) traversent la membrane basale en direction de gp 330 ( 9 ), une protéine membranaire associée à la clathrine $(T)$ dans les puits mantelés des pieds des podocytes. (B). Fixation des anticorps anti-gp 330 sur l'antigène induisant la formation d'un complexe immun membranaire. (C) Le complexe immun s'accroche à la membrane basale dès 15 minutes après l'injection de l'anticorps, et est relargué tout en restant en contact avec un puits mantelé. (D) Le dépôt immun grossit en raison de l'afflux permanent d'anticorps qui provoque la répétition du cycle d'événements décrit de (A) à (C) et empiète sur la zone du diaphragme. La formation des dépôts immuns requiert une synthèse de novo d'antigène gp 330 qui est véhiculé dans des vésicules jusqu'à la membrane cellulaire à la base des pieds des podocytes. 


\begin{tabular}{|c|}
\hline $\begin{array}{c}\text { Tableau ॥ } \\
\text { GLOMÉRULONÉPHRITES EXTRAMEMBRANEUSES HUMAINES : } \\
\text { CLASSIFICATION DES CAUSES POSSIBLES }\end{array}$ \\
\hline $\begin{array}{l}\text { GEM secondaires } \\
\text { Infections : hépatite } B^{*} \text {, syphilis, schistosomiase, filariose, hydatidose, strep- } \\
\text { tococcies, virus HIV (immunodéficience acquise) } \\
\text { Maladies systémiques : Iupus, connectivite mixte, polyarthrite rhumatoïde, } \\
\text { syndrome de Sjögren, dermatomyosite, sarcoïdose } \\
\text { Affections malignes : cancers* (poumon, côlon, estomac, sein...), lymphomes, } \\
\text { leucémies. } \\
\text { Toxiques et médicaments : sels d'or*, mercure*, D-pénicillamine }{ }^{*} \text {, trimétha- } \\
\text { dione, probénécide, captopril } \\
\text { Autres étiologies : drépanocytose, thyroïdite, pemphigoïde bulleuse, réaction } \\
\text { greffon contre hôte... } \\
\text { GEM idiopathiques } \\
\text { GEM de novo (sur greffon) }\end{array}$ \\
\hline
\end{tabular}

* Ces affections sont responsables de $75 \%$ des formes secondaires de GEM. cellules endothéliales glomérulaires a été suggéré par Jeraj et al. [24] au cours de la GNH passive et par Matsuo et al. [25] dans un modèle de GN induite chez le lapin par les anticorps dirigés contre l'enzyme de conversion de l'angiotensine (ACE). Chez la souris, gp 330 n'est pas exprimée par les cellules épithéliales glomérulaires et il est plus aisé de mettre en évidence le rôle de gp 90 dans un processus néphritogène. Assmann et $a l$. [26, 27] ont développé un modèle de GEM chez la souris, induit par injection d'anticorps de lapin fabriqués contre la bordure en brosse de souris digérée par la pronase (Tapron). La cinétique des dépôts glomérulaires est très proche de celle que nous avons observée chez le rat avec les anticorps anti-gp 90, mais la quantité d'anticorps qui persiste dans les glomérules est suffisante pour permettre le développement d'une phase autologue et d'une néphropathie glomérulaire chronique. En collaboration avec le groupe d'Assmann, nous avons pu montrer que les anticorps anti-Tapron utilisés contenaient des anticorps antigp 90 (DPP IV) et que ces anticorps étaient effectivement responsables de la GN observée. Des expériences récentes suggèrent que les anticorps anti-gp 90 pourraient être également impliqués dans la glomérulonéphrite extramembraneuse observée $\mathrm{m} / \mathrm{s} n^{\circ} 5$ vol. 5 , mai 89 servir de cible à un conflit immunologique. Ainsi, Izui et al. [30] ont montré que chez les souris lupiques, l'ADN circulant peut se fixer sur le collagène de la membrane basale glomérulaire et réagir in situ avec les anticorps correspondants. De nombreux exemples impliquant notamment des protéines cationiques (ferritine, IgG, sérumalbumine bovine [SAB]...) ont été rapportés et ont conduit à reconsidérer la physiopathologie des dépôts subépithéliaux dans la maladie sérique.

Dans la maladie sérique chronique [31] induite par l'administration répétée d'une dose constante d'antigène, la localisation des dépôts glomérulaires dépend beaucoup de l'amplitude de la réponse immune. Les animaux fort répondeurs ont des complexes immuns de grande taille qui ne se déposent pas dans les glomérules, les répondeurs intermédiaires ont des complexes plus petits et plus solubles et développent des dépôts mésangiaux et sous-endothéliaux, les faibles répondeurs, toujours en excès d'antigène, ont des complexes encore plus petits (300 à $500 \mathrm{kDa}$ ) associés à des dépôts extramembraneux. Si la dose d'antigène injectée est ajustée pour maintenir un état d'excès d'antigène, les dépôts sont localisés presque exclusivement sur le versant externe de la basale, quelle que soit l'amplitude de la réponse anticorps. Ces observations ont conduit à penser que les dépôts extramembraneux résultaient de la capacité des complexes immuns circulants de petite taille à traverser la membrane basale et à se localiser sur son versant externe. Cette hypothèse a été battue en brèche par les études portant sur la maladie du sérum passive montrant qu'il était impossible d'induire des dépôts extramembraneux par l'injection de complexes immuns préformés et par les expériences de perfusion de rein isolé avec alternativement l'antigène $(\mathrm{SAB}$ ou lysozyme) et l'anticorps aboutissant à la formation de dépôts subépithéliaux [32]. Ainsi, il est possible que, même dans la maladie sérique chronique, les dépôts extramembraneux résultent de la formation locale, dans la paroi capillaire, des complexes immuns. Ces faits doivent être gardés en mémoire dans la discussion des cibles antigéniques dans les GEM 
humaines. Ils suggèrent que les molécules cationiques sont de bonnes cibles potentielles, puisqu'elles sont susceptibles d'interagir avec les charges négatives du capillaire glomérulaire.

\section{Glomérulonéphrites extramembraneuses humaines}

Les GEM sont, avec les GN mésangiales à dépôts d'IgA (maladie de Berger), les glomérulopathies les plus fréquentes chez l'homme. Elles comptent parmi les causes principales de syndrome néphrotique. Elles conduisent à l'insuffisance rénale terminale dans 20 à $30 \%$ des cas. Elles sont observées dans des contextes pathologiques variés résumés dans le Tableau II. La multiplicité des causes possibles conduit à formuler deux questions essentielles: existe-t-il un ou plusieurs mécanismes de formation des dépôts extramembraneux chez l'homme? Existe-t-il une ou plusieurs antigènes néphritogènes ?.Il est aujourd'hui possible d'apporter quelques éléments de réponse.

Glomérunonéphrites impliquant des antigènes glomérulaires. En raison des similitudes importantes - cliniques, histologiques et immunologiques - entre les GEM humaines et la GN de Heymann chez le rat, on a cherché à mettre en évidence un mécanisme immunopathologique commun. Des antigènes tubulaires ont été identifiés dans les dépôts immuns dans des cas isolés de syndrome néphrotique associé à diverses affections (drépanocytose, cancer du rein, syndrome de Fanconi, thrombose de veines rénales) ainsi que dans quelques cas de GEM idiopathiques (revue dans [31]). Cependant, ces observations n'ont pas été confirmées. Le rôle des antigènes intrinsèques glomérulo-tubulaires ou glomérulaires reste très controversé. D'une part, il existe des arguments solides les disqualifiant: (1) les Ig éluées des glomérules des patients atteints de GEM ne réagissent qu'exceptionnellement avec le tissu rénal normal; (2) la perfusion de reins humains isolés non utilisés pour la transplantation avec des anticorps purifiés dirigés contre les antigènes formation de dépôts extramembraneux (Couser et Salant, observations non publiées). D'autre part, il existe des observations privilégiées dans lesquelles les dépôts extramembraneux sont associés à des dépôts tubulaires localisés sur la bordure en brosse. Cette discussion a amené le laboratoire de D. Kerjaschki [33] et le nôtre [34] à étudier l'expression de gp 330 chez l'homme. Cette protéine peut être détectée dans les régions intermicrovillaires de la bordure en brosse, mais elle est absente du glomérule (figure 1B). Le rat est, d'ailleurs, la seule espèce dans laquelle gp 330 est exprimée dans les puits mantelés des cellules épithéliales glomérulaires. De plus, il n'a pas été possible d'identifier gp 330 dans les dépôts immuns glomérulaires de 30 biopsies de GEM. Ces observations plaident contre la responsabilité de gp 330 dans les GEM humaines sans toutefois l'exclure. Il est possible, en effet, que la protéine soit synthétisée très faiblement dans les podocytes normaux, et de façon plus active après l'initiation du conflit immunologique, à la surface de la cellule [19]. D'autre part, Makker et Kanalas [35] ont récemment mis en évidence des Ac antigp 330 dans le sérum de patients atteints de GEM.

Les problèmes soulevés par gp 90 (la dipeptidyl peptidase IV) et l'endopeptidase neutre (figure 6) sont différents. Contrairement à gp 330, ces deux protéines enzymatiques sont présentes de façon diffuse sur la mem-

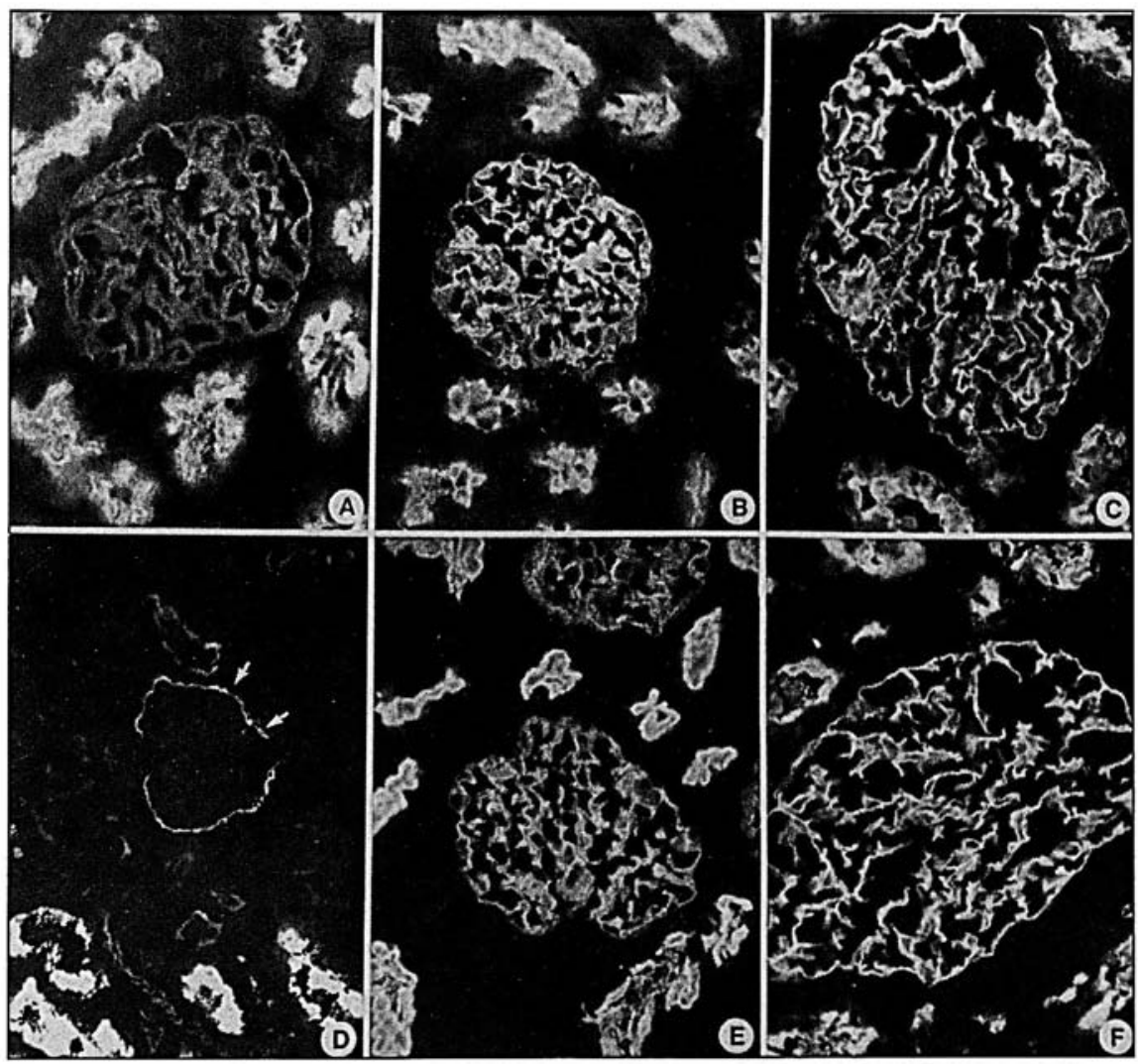

Figure 5. Expression comparative de la dipeptidyl peptidase IV (A-C) et de l'endopeptidase neutre (D-F) chez le rat (A, D), le lapin (B, E) et I'homme (C, F), étudiée en immunofluorescence indirecte sur des coupes à congélation de cortex rénal incubées avec les anticorps monoclonaux correspondants. Noter que la dipeptidyl peptidase $N$ a une distribution comparable dans les trois espèces sur la bordure en brosse et le long des parois des capillaires glomérulaires. L'endopeptidase neutre a une localisation similaire chez le lapin et l'homme. En revanche, chez le rat, elle n'est exprimée que par les segments S3 des tubes contournés proximaux localisés dans le cortex profond (partie inférieure de la photographie) et par les cellules de la capsule de Bowman (flèche); les segments S1 et S2 des tubes contournés proximaux dans le cortex superficiel sont négatifs (partie supérieure de la photo). (x 250.) 


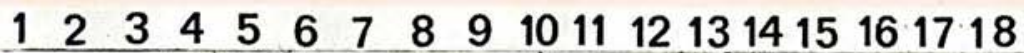

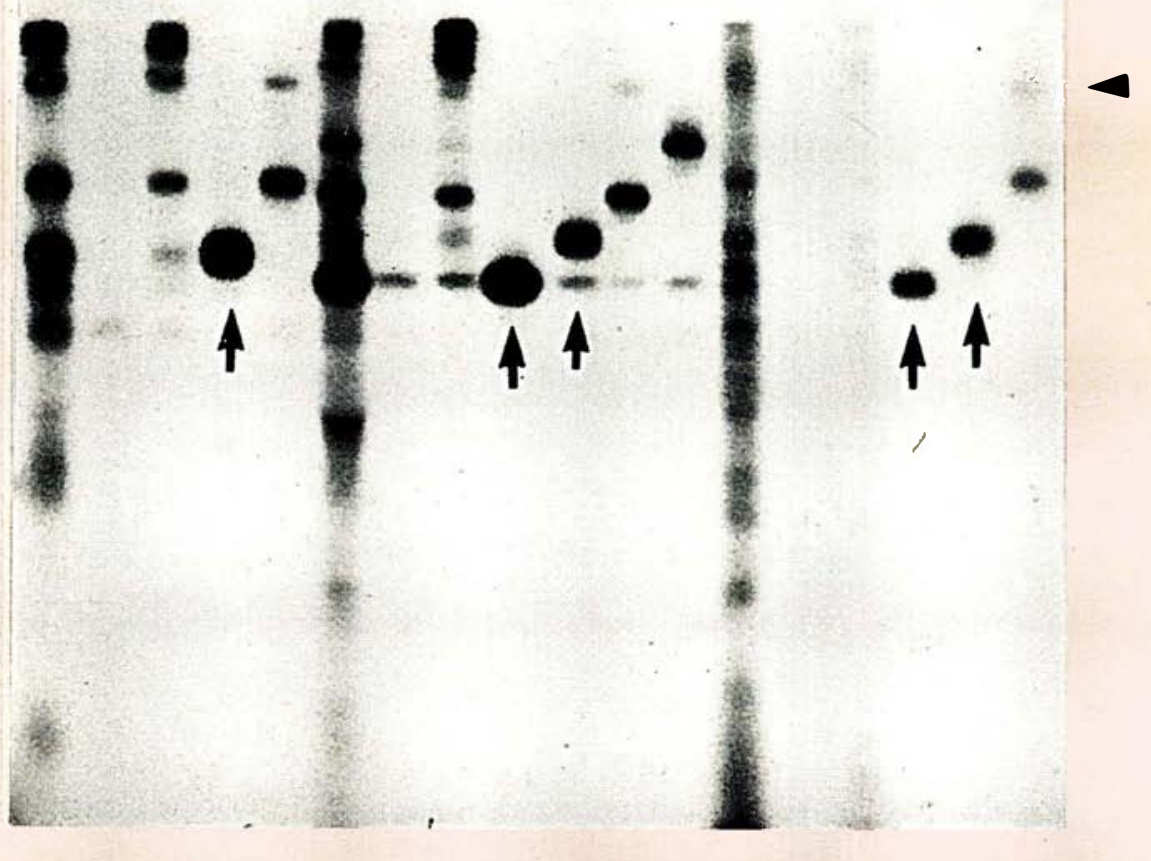

Figure 6. Comparaison des poids moléculaires (PM) apparents des antigènes de bordure en brosse du rat (lignes 13-18) identifiés par des anticorps monoclonaux produits spécifiquement contre la bordure en brosse des trois espèces. Les protéines de la bordure en brosse sont marqués à l'iode ${ }^{125}$, immunoprécipitées par les différents anticorps et appliqués sur un gel de polyacrylamide en présence de SDS, dans lequel la migration se fait essentiellement en fonction du PM. Les marqueurs de PM (têtes de flèche) sont, de haut en bas, I'lgG, 150 kDa, et la chaîne $\mu$ de l'IgM, $80 \mathrm{kDa}$. Lignes 1, 6, 13 : protéines totales de la bordure en brosse; lignes 2, 7, 14 : immunoprécipitation témoin réalisée avec le sérum de souris normale; lignes 3, 8, 15 : immunoprécipitation effectuée avec les sérums des souris immunisées avec la bordure en brosse et utilisées comme pourvoyeurs de splénocytes pour la fusion cellulaire; lignes 4, 10, 17: la bande immunoprécipitée est la dipeptidyl peptidase $N$, dont le PM apparent est légèrement plus faible chez le rat (90 kDa) que dans les deux autres espèces (100 kDa); lignes 5, 11, 18 : leucine aminopeptidase (115 kDa); lignes 9 et 16 : endopeptidase neutre ( $85 \mathrm{kDa}$ ) identique chez l'homme à l'antigène commun des leucémies aiguës lymphoblastiques (Calla); ligne 12: enzyme de conversion de l'angiotensine $(140 \mathrm{kDa})$. Les flèches indiquent des protéines exprimées à la fois sur la bordure en brosse et les cellules épithéliales glomérulaires. Noter la conservation phylogénique des protéines de bordure en brosse les plus immunogènes.

brane des cellules épithéliales glomérulaires humaines (figures $5 C$ et $5 F$ ). Leur localisation a été étudiée grâce à des anticorps monoclonaux produits initialement contre la bordure en brosse de rat ou de lapin et dotés d'une réactivité croisée avec le rein humain, et à une nouvelle famille d'anticorps monoclonaux spécifiquement dirigés contre la bordure en brosse humaine [34]. En collaboration avec Michelle Letarte et al. [36], nous avons récemment montré l'identité antigénique de l'endopeptidase neutre et de l'antigène commun des leucémies aiguës lymphoblastiques (Calla), qui sont codées par le même gène. Les deux antigènes sont impliqués chez l'animal $\mathrm{m} / \mathrm{s} n^{\circ} 5$ vol. 5 , mai 89 dans la formation de dépôts extramembraneux transitoires, dont la contrepartie chez l'homme pourrait être les GEM médicamenteuses. En effet, celles-ci sont remarquables par leur régression très rapide à l'arrêt du médicament. Cette évolution est particulièrement caricaturale dans le cas des sels d'or et de la D-pénicillamine [37, 38]. Il est intéressant de noter que certains de ces médicaments ont une toxicité tubulaire qui pourrait favoriser la libération dans la circulation des antigènes tubulaires et le déclenchement d'un processus autoimmun.

D'autres antigènes glomérulaires non identifiés sont des cibles potentielles. Des observations récentes sug- gèrent que certains anticorps antiADN réagissent avec des protéines glomérulaires et peuvent induire la formation de dépôts immuns [39]. Glomérunonéphrites impliquant des antigènes non glomérulaires. Un certain nombre d'antigènes non glomérulaires ont été identifiés dans les dépôts extramembraneux chez l'homme (revue dans [31]), qu'il s'agisse de l'ADN, des antigènes $\mathrm{HBs}$ et $\mathrm{HBe}$, d'antigènes thyroïdiens et tumoraux, et tout récemment de l'antigène P24 du noyau du virus HIV1 dans deux cas de GEM (Rao, communication personnelle). Si les études les plus anciennes effectuées à l'aide d'anticorps polyclonaux pouvant manquer de spécificité prêtent à 


\section{RÉFÉRENCES}

(GEC) are target antigens for the formation of immune depotits (ID). Kidney Int 1987 ; 31 : 329 (abstr.).

29. Tauc M, Chatelet F, Verroust $P$, Vandewalle A, Poujeol PH, Ronco P. Characterization of monoclonal antibodies specific for rabbit renal brush border hydrolases: application to immunohistological localization. $J$ Histochem Cytochem 1988 ; 35 : 523-32.

30. Izui S, Lambert PH, Miescher PA. In vitro demonstration of a particular affinity of glomerular basement membrane and collagen for DNA : a possible basis for focal formation of DNA-anti DNA-complexes in systemic lupus erythematosus. J Exp Med 1976; 144 : 428-43.

31. Wilson CB, Dixon JF: Renal response to immunological injury. In : Brenner BM, Rector FC, eds. The Kidney. Philadelphia: WB Saunders 1986 : 800-89.

32. Fleuren G, Grond J, Hoedemaeker PJ. In situ formation of subepithelial glomerular immune complexes in passive serum sickness. Kidney Int 1980; 17 : 631-7.

33. Kerjaschki D, Horvat R, Binder S, et al. Identification of a $400 \mathrm{kDa}$ protein in the brush borders of human kidney tubules that is similar to gp 330, the nephritogenic antigen of rat Heymann nephritis. Am J Pathol 1987 ; 129 : 183-91.

34. Geniteau M, Melcion C, Ronco P, Brunisholz M, Verroust P. Production of monoclonal antibodies against constituents of the renal tubule in human kidney. Eur J Clin Invest 1984 ; 14 : 60 (abstr.).

35. Kanalas JJ, Makker SP. Isolation of $330 \mathrm{kDa}$ human kidney protein similar to the rat Heymann nephritis autoantigen (gp 330). Kidney Int 1989 ; 35 : 351 (abstr).

36. Jongeneel CV, Quackenbush EJ, Ronco $P$, Verroust P, Carrel S, Letarte M. The common acute lymphoblastic leukemia antigen (Calla) expressed on leukemia and melanoma cell lines has neutral endopeptidase activity. J Clin Invest 1989 ; 83 : 713-7.

37. Hall CL, Fothergill NJ, Blackwell MM Harrison PR, MacKenzie JC, MacIver AG. The natural course of gold nephropathy: long term study of 21 patients. Br Med J 1987 ; $295: 745-8$.

38. Hall CL, Jawad S, Harrison PR, et al. Natural course of penicillamine nephropathy : a long term study of 33 patients. $\mathrm{Br}$ Med J 1988 ; 296 : 1083-6.

39. Jacob L, Tron F, Bach JF, Louvard D. A monoclonal anti-DNA antibody also binds to cell-surface protein(s). Proc Natl Acad Sci discussion, les plus récentes échappent à cette critique car elles utilisent des anticorps monoclonaux. Cependant, les résultats doivent être nuancés en tenant compte de la perméabilité accrue de la membrane basale qui favorise le piégeage non spécifique des molécules dans les dépôts immuns. Les mécanismes qui aboutissent à la formation de ces dépôts sont controversés, et la discussion reste ouverte entre le dépôt de complexes immuns circulants et la formation de complexes in situ. Il est possible que les deux mécanismes coexistent. Il est très vraisemblable que les dépôts, une fois constitués, servent à leur tour de cible antigénique pour des facteurs rhumatoïdes ou des anticorps anti-idiotypiques.

\section{Stratégies pour l'avenir}

L'identification des principaux antigènes néphritogènes impliqués dans les GEM humaines est vraisemblable dans la prochaine décennie. L'approche monoclonale utilisée dans les modèles expérimentaux et par nousmêmes chez l'homme doit être répétée, en utilisant comme matériel immunogène des préparations membranaires de glomérules isolés. Les anticorps monoclonaux produits seront caractérisés par leur spécificité antigénique et, éventuellement, leur pouvoir pathogène chez l'animal. Ils seront utilisés pour analyser les dépôts extramembraneux en utilisant les techniques d'immunofluorescence et d'immunomicroscopie électronique, à la recherche d'une concentration des antigènes intrinsèques glomérulaires dans les dépôts. De plus, l'analyse de la spécificité d'éluats glomérulaires précoces, préparés à la phase initiale de la GEM avant que les dépôts ne soient remaniés, devrait permettre de déterminer la part respective des antigènes glomérulaires intrinsèques et des antigènes étrangers au glomérule dans la pathogénie des GEM humaines. Sur le plan thérapeutique, l'identification des cibles antigéniques pourrait déboucher sur l'utilisation d'immuno-adsorbants spécifiques permettant d'éliminer les anticorps dirigés contre les antigènes néphritogènes

\section{Summary}

Antigenic targets in epimenbranous glomerulonephritis

More than twenty years after the first immunofluorescence studies of renal biopsies showing that most human glomerulonephritis (GN) contained immune deposits, the antigenic targets involved remain most often unidentified. Studies of experimental models of membranous glomerulonephritis (MGN) have permitted significan advances in this field. The antigen responsible for Heymann's MGN induced by immunization of rat with proximal tubule brush border antigens has been identified as gp 330 , a $330 \mathrm{kDa}$ protein restricted to coated pits of the brush border and the glomerular epithelial cells. Formation of subepithelial electron dense deposits characteristic of MGN involves clustering of multivalent gp 330-anti-gp 330 complexes in the coated pits facing the glomerular capillary wall followed by rapid adherence of these complexes to basement membrane structures. Following a strategy that consisted in raising monoclonal antibodies against the brush border and selecting those reacting with the glomerulus, we identified two additional antigenic specificities endowed with dipeptidyl peptidase IV (DPP IV) and neutral endopeptidase (NEP) activity and implicated in the formation of shortlived or long-lasting subepithelial deposits in mice, rats and rabbits. Other «nephritogenic» antigens include nonglomerular circulating antigens planted in the glomerular capillary wall because of cationic charge or affinity for basement membrane constituents. Human cases of MGN are heterogeneous with respect to evolution and etiology and also probably to target antigens and mechanisms of immune deposit formation. Among previously described antigens, gp 330 is not a good candidate because it is not expressed in human glomeruli. In contrast, DPP IV and NEP (recently identified as Calla) might be involved in the pathogenesis of drug-induced short-lived MGN. Nonglomerular antigens detected in subepithelial deposits, such as microbial antigens, might be deposited either first as planted antigens which then bind in situ to circulating antibodies or simultaneously with the corresponding antibodies within low molecular weight circulating immune complexes passively trapped in the glomerular capillary wall. 